\title{
Platelet Endothelial Cell Adhesion Molecule (PECAM-1) Expression in Malignant Human Tumours and their Metastases
}

\author{
Juan R de la Haba Rodríguez, ${ }^{1}$ Ignacio Porras Quintela ${ }^{1}$, Gema Pulido Cortijo ${ }^{1}$, Concepción Lucena \\ Martínez $^{2}$, Javier García Corbacho ${ }^{1}$, Enrique Aranda Aguilar ${ }^{1}$ \\ ${ }^{1}$ Medical Oncology and Pathology Departments, Reina Sofía University Hospital, Córdoba, Spain; ${ }^{2}$ Department of Cell Biology, \\ Physiology and Immunology, University of Cordoba, Córdoba, Spain. \\ Email: juandelahaba@hotmail.com
}

Received April 16 $6^{\text {th }}, 2010$; revised May $21^{\text {st }}, 2010$; accepted May $28^{\text {th }}, 2010$

\begin{abstract}
PECAM-1 is an adhesion molecule that plays an important role in the process of tumour disease dissemination since a function in transendothelial migration, angiogenesis and immune response has been shown for this membrane protein. Nevertheless the expression of PECAM-1 protein in solid tumours is a controversial matter and it has not been clarified so far. Thus, the aim of our study was to investigate PECAM-1 expression by immunohistochemistry in primary carcinomas from colon, breast, bladder, ovary and kidney, and in their metastases. In addition an example of primary and metastatic melanoma was also investigated. We found that PECAM-1 is expressed in the metastases of all primary carcinomas that express PECAM-1 (colorectal, breast and urothelial bladder). By the contrary metastases from primary carcinomas non-expressing PECAM-1 are also negative for expression. In conclusion, our findings support a possible role of this molecule in metastatic development of a subset of malignant human epithelial tumours.
\end{abstract}

Keywords: PECAM-1, CD31, Angiogenesis, Neoplasms, Immunohistochemistry

\section{Introduction}

Metastatic dissemination is a complex biological process in which the tumoral cells escape immunological surveillance, migrate from its initial site through the vascular endothelium and finally grow up into a new tissue. The expression of adhesion molecules plays a key role in the process of metastases and neoangiogenesis.

PECAM-1 (CD31 or EndoCAM), a member of the immunoglobulin superfamily, is a membrane glycoprotein type 1 of 711 aminoacids [1] classified as an adhesion molecule [2]. It is a member of the inmunoglobulin super family that is encoded by a $75-\mathrm{kb}$ gene that resides at the end of the long arm of chromosome 17 [3]. This glycoprotein is expressed in platelets, lymphocytes, monocytes, natural killer cells [4,5] and also in interendothelial junctions[6]. PECAM-1 has either homophilic $[7,8]$ or heterophilic adhesion capacities with other molecules such as integrines $[9,10]$ and glycosaminoglycanes [11]. Moreover, PECAM-1 trough its cytoplasmic domain transmit out-in signals from the cell surface to the nucleus $[12,13]$. In experimental inflammation mod- els a functional inhibition of PECAM-1 with monoclonal antibodies prevents leukocyte migration to the inflammatory focus $[14,15]$. In addition, PECAM-1 also participates in the T-cell mediated activation in alloimmune response [16] and in natural killer cell activation, proliferation and migration [17]. Recent studies have demonstrated an even wider range of functions for CD31 including maintenance of adherens junction integrity and permeability, organization of the cytoskeleton, transcriptional activities, participation in STAT isoform signaling among others. [18]

PECAM-1 is also believed that participate in the neoangiogenesis process $[6,19]$. For instance, it has been suggested that PECAM-1 play an important role in the formation of new vessels, through homophilic interactions in the endothelial junctions, or the heterophilic adhesion the integrine $\alpha_{\mathrm{v}} \beta_{3}$, which are crucial unions in endothelial cell migration through the extracellular matrix. [20]. To gain insight into the role PECAM-1 plays during vascular development and angiogenesis, for example it was examined the expression pattern of PECAM-1 isoforms during kidney vascularization showing 
that regulated expression of specific PECAM-1 isoforms may enable endothelial cells to accommodate the different stages of angiogenesis [21].

PECAM-1 has been shown to potently suppress apoptosis in a variety of cellular systems, for example on a variety of human malignancies -especially hematopoietic and vascular cell cancers-. The ability of PECAM-1 to inhibit apoptosis makes it an attractive candidate as a molecule that may promote cancer development and/or confer resistance to chemotherapeutic treatment. In a recent study, it was shown that the endogenous PECAM-1 expression on lymphoid cancers confers resistance to apoptosis, and that lowering PECAM-1 expression in lymphoid malignancies can render them more susceptible to chemotherapy-induced apoptosis. [22,23]. The expressión of PECAM-1 in some hematopoietic malignancies has been studied and correlated with a worse prognosis, for example in a subgroup of patients with B-cell chronic lymphocytic leukemia (B-CLL) [24] or with primary non-Hodgkin's gastric lymphoma. [25].

Previous studies have shown that some cellular lines derived from solid tumours express PECAM-1 (Hep-1, MS751, TCC, MFC-7, DLD-1) and that anti-PECAM-1 monoclonal antibodies inhibit the tumoral cell adhesion to the endothelium "in vitro" [26]. Interestingly, it has been observed that in gliomas [27], breast carcinomas, osteosarcomas and in lymphomas [28] PECAM-1 expression is related to spread and disease progression. Moreover, PECAM-1 has also been used as a histological neoangiogenesis marker since a correlation between expression and prognosis has been demonstrated in breast cancer and melanomas [29,30]. The expression of PE-
CAM-1 could be related to endothelial transdifferentiation of melanoma cells although a consequent functional role has not been demonstrated yet [31].

The functions in which PECAM-1 may participate (adhesion, transendothelial migration and neoangiogenesis) are key steps in metastatic dissemination. Therefore the study of PECAM-1 in primary and metastatic tumours is of special interest to understand the role of this antigen in the metastatic process. In the present report we have analysed PECAM-1 expression in a selected group of common solid human tumours both in primary and in metastatic localisation.

\section{Material and Methods}

Tissue samples from colon, breast, urinary bladder and kidney carcinomas and melanoma were selected to study the expression of PECAM-1 (Table 1). Surgical material was fixed in $4 \%$ phosphate-buffered formalin and paraffin embedded.

Selected 4-6 mms sections were utilized for immunohistochemistry using the avidin-biotin-peroxidase complex method. After deparaffination, unstained slides were treated with microwave heating for antigen retrieval solution (citrate buffer $\mathrm{pH}$ 6.0) for 12 minutes. The antiPECAM-1 antibody (clone HC 1/6) (Cabañas, 1989) was applied at a 1:1 dilution, then followed by biotinilated anti-mouse Ig G secondary antibody and Vectastain ABC kit (Vector Laboratories Inc.). Diaminobenzidine (Sigma) was used as substrate, and the slides were slightly counterstained with hematoxylin. Negative controls were carried out in the absence of the specific antibody and

Table 1. Immunohistochemical expression of PECAM-1 in colon cancer, breast cancer and bladder cancer specimens

\begin{tabular}{|c|c|c|c|c|}
\hline Identification & $\begin{array}{l}\text { Localisation } \\
\text { Tumour }\end{array}$ & $\begin{array}{c}\text { Immunohisto-chemical } \\
\text { expression }\end{array}$ & $\begin{array}{c}\text { Localisation } \\
\text { Metastases }\end{array}$ & $\begin{array}{c}\text { Immunohisto-chemical } \\
\text { expression }\end{array}$ \\
\hline FCH5388/5342 & Colorectal & +++ & Liver & ++ \\
\hline CMM670/671 & Colorectal & ++ & Liver & ++ \\
\hline MRL6638/6640 & Colorectal & +++ & Liver & +++ \\
\hline ARM17997/17994 & Colorectal & +++ & Liver & +++ \\
\hline JRR11842/11003 & Colorectal & + & Liver & ++ \\
\hline VFR13009/14142 & Breast & +++ & Bone & + \\
\hline FFS4514/16987 & Breast & ++ & Skin & +++ \\
\hline AUV4646/16954 & Ovary & - & Skin & - \\
\hline RMH18421/2795 & Urinary bladder & ++ & Lung & ++ \\
\hline CCR12186/8921 & Melanoma & - & Bone & - \\
\hline PLP9529/10689 & Kidney & - & Skin & - \\
\hline
\end{tabular}

Quantitation of immunostaining in the tumours: $(-): 0 \% ;(+): 1-10 \% ;(++): 11-50 \% ;(+++):>50 \%$. 
the vascular-endothelial tissue area was the positive control that serve us to quantified PECAM-1 expression in tumoral cells (Figure 1). Quantitation of immunostaining in the tumours was semiquantitatively assessed as (-): $0 \%$; $(+)$ : $1-10 \%$; $(++): 11-50 \%$; $(+++):>50 \%$. The staining evaluation was assessed independently by two experimented pathologists.

\section{Results}

Immunohistochemical expression of PECAM-1 gave positive results in colon cancer, breast cancer and bladder cancer specimens (Figure 2). With the exception of bladder cancer the immunostaining for PECAM-1 was heterogeneous throughout the tumour sample with membranous and intracytoplasmic pattern.

As seen in Table 1, the immunostaining for PECAM-1 was positive in all colon, breast and urinary bladder cancers with different degrees of expression. In general, the expression was slightly more reduced in metastasic than in primaries tumours samples, with the exception of one of the colon cancer cases, which showed increased expression of the marker in the metastatic localisation (Figure 3). Immunohistochemical expression of PECAM-1 was not related to tumour grade or stage but

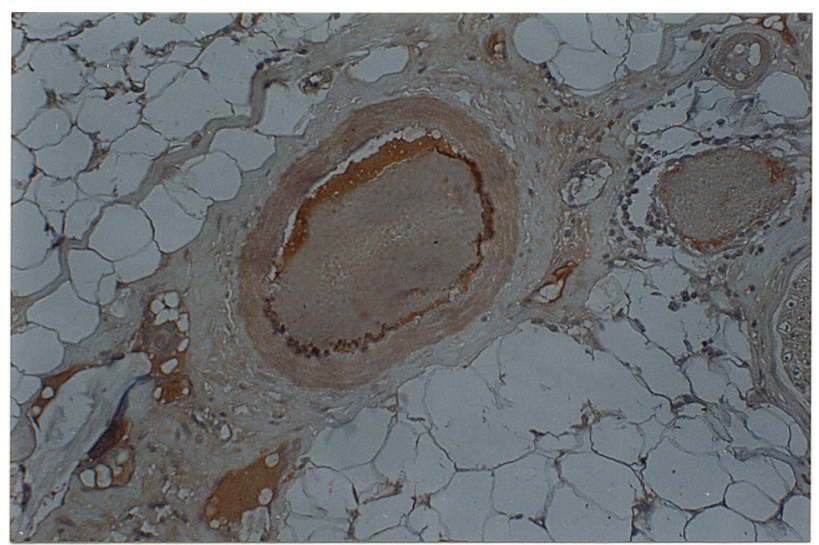

Figure 1. Tumoral Endothelial expression on PECAM-1

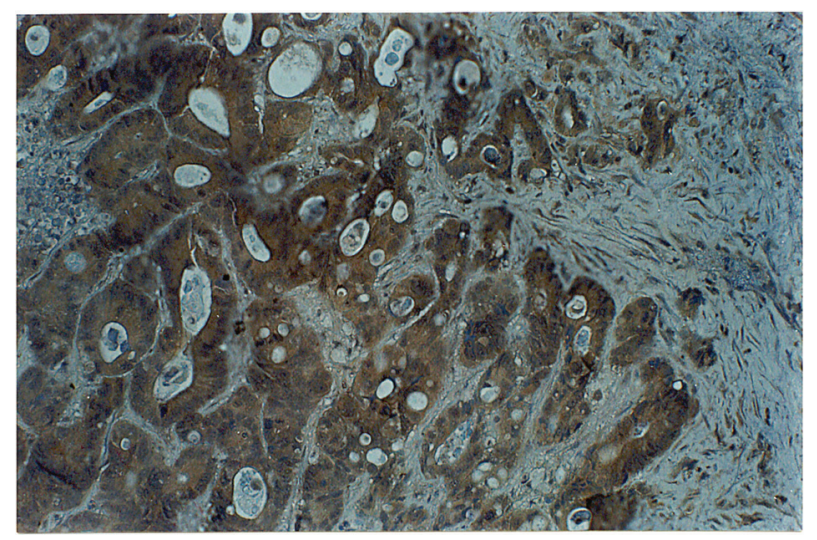

Figure 2. Expression of PECAM-1 in colorectal cancer

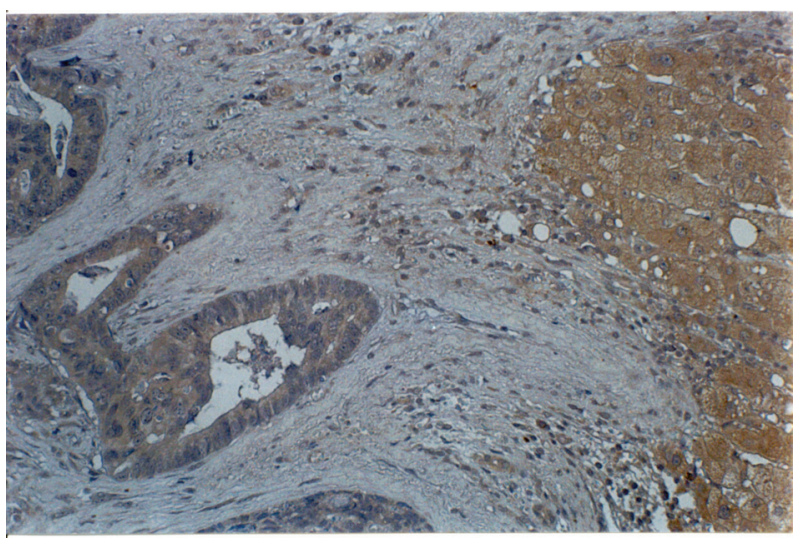

Figure 3. Expression of PECAM-1 in liver metastases of colorectal cancer

interestingly in bladder cancer the expression of this protein was limited to the most basal layers.

Melanoma and ovarian or kidney carcinomas did not show any immunohistochemical expression of PECAM-1 either in primary or metastatic sites.

\section{Discussion}

Although PECAM-1 expression has been studied in cellular lines of solid tumours, leukemias and lymphomas to the best of our knowledge the expression of this adhesion protein has not been evaluated in human tumours. Thus, the aim of the present study is to show this expression and the correlation between primary and metastatic localisation. This is important since it can strongly suggests that PECAM-1 may have a significant role in the metastatic process of some epithelial tumours, mainly colon cancer, breast cancer and probably bladder cancer and it could represent a different therapeutic approach by blocking PECAM-1 in some type of human cancers.

At the present we don't known whether the PECAM-1 expression in these tumours is constitutive or regulated by external stimuli. An interesting observation is the heterogeneous expression of PECAM-1 through the tumoral tissue in bladder samples. This can be explained by the heterogeneous character of neoplasms but it can also be argued that PECAM-1 expression identifies cellular clones which are more able to metastasize in epithelial tumours. Notwithstanding, PECAM-1 has been shown to be up-regulated by inflammatory cytokines (Rival, 1996; Romer, 1995) and the promoter region of this gene, which has been identified recently, contains sites responsive to transcription factors activated in the processes of cellular differentiation and proliferation (Almedro, 1996). Moreover, a functional site for the NF-kB factor has been also identified (Botella, 2000). It is well known that this transcription factor is stimulated by a wide range of external stimuli including mechanical factors and it is possible that cellular subgroups, located mainly at the basal cells layers, with higher proliferating activity express 
PECAM-1 by means of the activation of transcription factor NF-kB. We are currently studying the possible correlation between PECAM-1 expression and NF-kB nuclear localisation in different areas of selected tumoral tissues.

Very little is known at present with respect to the function that PECAM-1 may have in tumour cells "in vivo", although some studies, as the one presented here, have suggested a possible role in metastatic spreading. Thus, it is possible that solid neoplasm expressing PECAM-1 are endowed with a greater capacity to migrate and disseminate. Nevertheless, we observed that some disseminated tumours do not show PECAM-1 expression and this result point out that the possible role of PECAM-1 in the tumoral cells is not universal, although the function of PECAM-1 in angiogenesis is well documented even in PECAM-1 negative tumours. Further studies are required to determine the important of PECAM-1 in the whole process of tumour progression as well as the molecular mechanisms that regulated the expression of this adhesion protein in the primary tumour.

\section{REFERENCES}

[1] H. Stockinger, S. J. Gadd, R. Eher, O. Madjdic, et al., "Molecular Characterization and Functional Analysis of the Leukocyte Surface Protein CD31," Journal of Immunology, Vol. 11, 1990, pp. 3889-3897.

[2] P. Newman, M. Berndt, J. Gorski, et al., "PECAM-1 (CD31) Cloning and Relation to Adhesion Molecules of the Immunoglobulin Gene Superfamily," Science, Vol. 247, 1990, pp. 1219-1222.

[3] P. Newman, "The Biology of PECAM-1," Journal of Clinical Investigation, Vol. 99, No. 1, 1997, pp. 3-8.

[4] P. Newman and S. Albelda, "Cellular and Molecular Aspects of PECAM-1," Nouv Rev Fr Hematol, Vol. 34, Supplement S9-13, 1992.

[5] L. Piali, B. Imhof and R. Gisler, "Adhesion Molecule PECAM-1/CD31 Is Expressed on Defined Subsets of Murine LAK Cells," Advances in Experimental Medicine and Biology, Vol. 355, 1994, pp. 147-153.

[6] S. Albelda, "Endothelial and Epithelial Cell Adhesion Molecules," American Journal of Respiratory Cell and Molecular Biology, Vol. 4, 1991, pp. 195-203.

[7] S. Albelda, W. Muller, C. Buck and P. Newman, "Molecular and Cellular Properties of PECAM-1 (Endocam/CD31): A Novel Vascular Cell-Cell Adhesion Molecule," Journal of Cell Biology, Vol. 114, 1991, pp. 10591068.

[8] J. C. Fawcett, C. L. Buckley, I. N. Holness, J. H. Bird, J. Spragg and A. Sauders, "Mapping the Homotipic Binding Sites in CD31 and the Role of CD31 in the Formation of Interendothelial Cells Contacts," Journal of Cell Biology, Vol. 128, 1995, pp. 1229-1241.

[9] L. Piali, P. Hammel, C. Uherek, et al., "CD31/PECAM-1 Is a Ligand for Alpha V Beta 3 Integrin Involved In Ad- hesion of Leukocytes to Endothelium," Journal of Cell Biology, Vol. 130, 1995, pp. 451-460.

[10] R. Chiba, N. Nakagawa, K. Kurasawa, Y. Tanaka, Y. Saito and I. Iwamoto, "Ligation of CD31 (PECAM-1) on Endothelial Cells Increases Adhesive Function of Alphavbeta3 Integrin and Enhances Beta1 IntegrinMediated Adhesion of Eosinophils to Endothelial Cells," Blood, Vol. 94, 1999, pp. 1319-1329.

[11] H. DeLisser, H. Yan, P. Newman, W. Muller, C. Buck, S. Albelda, "Platelet/endothelial Cell Adhesion Molecule-1 (CD31)-Mediated Cellular Aggregation Involves Cell Surface Glycosaminoglycans," Journal of Biological Chemistry, Vol. 268, 1993, pp. 16037-16046.

[12] M. Streuli, "Protein Tyrosine Phosphatases in Signalling," Current Opinion in Cell Biology, Vol. 8, 1996, pp. 182-188.

[13] N. Ilan, L. Cheung, E. Pinter and J. A. Madri, "PlateletEndothelial Cell Adhesion Molecule-1 (CD31), a Scaffolding Molecule for Selected Catenin Family Members Whose Binding Is Mediated by Different Tyrosine and Serine/Threonine Phosphorylation," Journal of Biological Chemistry, Vol. 275, 2000, pp. 21435-21443.

[14] S. Bogen, J. Pak, M. Garifallou, X. Deng and W. Muller, "Monoclonal Antibody to Murine PECAM-1 (CD31) Blocks Acute Inflammation in Vivo," Journal of Experimental Medicine, Vol. 179, 1994, pp. 1059-1064.

[15] A. Vaporciyan, H. DeLisser, H. Yan, et al., "Involvement of Platelet-Endothelial Cell Adhesion Molecule-1 in Neutrophil Recruitment in Vivo," Science, Vol. 262, 1993, pp. 1580-1582.

[16] J. L. Zehnder, M. Shatsky, L. L. K. Leung, E. C. Batcher, et al., "Involvement of CD31 in Lymphocyte Mediated Inmune Responses: Importance of the Membrane Proximal Immunoglobuline Domain and Identification of an Inhibiting CD31 Peptide," Blood, Vol. 85, 1995, pp. 1282-1288.

[17] M. E. Berman, Y. Xie, W. A. Muller, "Roles of PECAM-1 in Natural Killer Cell Trans Endothelial Migration and Beta-2 Integrin Activation," Journal of Immunology, Vol. 156, 1996, pp. 1515-1524.

[18] V. Lutzky, R. Carnevale, M. Alvarez, P. Maffia, S. Zittermann, O. Podhajcer. et al., "Platelet-Endothelial Cell Adhesion Molecule-1 (CD31) Recycles and Induces Cell Growth Inhibition on Human Tumor Cell Lines," Journal of Cell Biochemistry, Vol. 98, 2005, pp. 1334-1350.

[19] H. DeLisser, M. Christofidou-Solomidou, R. Strieter, et al., "Involvement of Endothelial PECAM-1/CD31 in Angiogenesis," American Journal of Pathology, Vol. 151, 1997, pp. 671-677.

[20] J. Bischoff, "Cell Adhesion and Angiogenesis," Journal of Clinical Investigation, Vol. 99, 1997, pp. 373-376.

[21] S. Kondo, E. Scheef, N. Sheibani and C. Sorenson, "PECAM-1 Isoform-Specific Regulation of Kidney Endothelial Cell Migration and Capillary Morphogenesis," American Journal of Physiology, Vol. 292, 2007, pp. 2070-2083.

[22] C. Bergom, C. Gao and P. Newman, "Mechanisms of 
PECAM-1-Mediated Cytoprotection and Implications for Cancer Cell Survival," Leuk Lymphoma, Vol. 46, No. 10, 2005, pp. 1409-1421.

[23] C. Bergom, R. Goel, C. Paddock, C. Gao, D. K. Newman, S. Matsuyama and P. J. Newman, "The Cell-Adhesion and Signaling Molecule PECAM-1 Is a Molecular Mediator of Resistance to Genotoxic Chemotherapy," Cancer Biology \& Therapy, Vol. 5, No. 12, 2006, pp. 1699-1707.

[24] S. Ibrahim, I. Jilani, S. O’Brien, A. Rogers, T. Manshouri, F. Giles, et al., "Clinical Relevance of the Expression of the CD31 Ligand for CD38 in Patients with B-Cell Chronic Lymphocytic Leukemia," American Cancer Society, Vol. 97, No. 8, 2003, pp. 1914-1919.

[25] A. Darom, I. Gomatos, E. Leandros, E. Chatzigianni, D. Panousopoulos, Konstadoulakis and G. Androulakis, "Molecular Markers (PECAM-1, ICAM-3, HLA-DR) Determine Prognosis in Primary Non-Hodgkin's Gastric Lymphoma Patients," World Journal of Gastroenterology, Vol. 12, No. 12, 2006, pp. 1924-1932.

[26] D. Tang, Y. Chen, P. Newman, et al., "Identification of PECAM-1 in Solid Tumour Cells and Its Potential Involvement in Tumour Cell Adhesion to Endothelium," Journal of Biological Chemistry, Vol. 268, 1993, pp.
22883-22894.

[27] F. Aroca, W. Renaud, C. Bartoli and C. Bouvier-lavit, "Expression of PECAM-1/CD31 Isoforms in Human Brain Gliomas," Journal of Neuro-Oncology, Vol. 43, 1999, pp. 19-25.

[28] E. Roos, "Adhesion Molecules in Lymphoma Metastasis," Cancer and Metastasis Reviews, Vol. 10, 1991, pp. 33-48.

[29] S. Fox, G. Turner, R. Leek, R. Whitehouse, K. Gatter and A. Harris, "The Prognostic Value of Quantitative Angiogenesis in Breast Cancer and Role of Adhesion Molecule Expression in Tumour Endothelium,"Breast Cancer Research and Treatment, Vol. 36, 1995, pp. 219-226.

[30] T. Vlaykova, P. Laurila, T. Muhonen, M. Hahka- kemppinen, et al., "Prognostic Value of Tumour Vascularity in Metastatic Melanoma and Association of Blood Vessel Density with Vascular Endothelial Growth Factor Expression," Melanoma Research, Vol. 9, 1999, pp. 59-68.

[31] A. Pisacane, F. Picciotto and M. Risio, "CD31 and CD34 Expression as Immunohistochemical Markers of Endothelial Transdifferentiation in Human Cutaneous Melanoma," Cellular Oncology, Vol. 29, 2007, pp. 59-66. 